violet data from the Orbiting Astronomical Observatory 2 (Holm Astrophys. J. Lett. 210, L87) imply that Feige 24 is not quite so hot, around 6 to $7 \times 10^{4} \mathrm{~K}$. To predict the EUV fluxes, Margon et al. attempt a model atmosphere calculation for a pure hydrogen atmosphere, and they find that the low observed flux shortward of $170 \AA \mathrm{im}$ plies that there must be at least some helium present in the atmosphere to provide enough opacity shortward of the ionised helium edge. They suggest a possible, but less probable, alternative in the presence of absorption by ionised helium in the interstellar gas. Feige 24 is known from spectroscopic evidence to be a binary pair of a white dwarf and a cool main sequence dwarf showing emission lines. By subtracting the expected infrared flux of the white dwarf from the total flux of the pair, Holm deduces the energy distribution and hence a rough classification for the main sequence star. By comparison with known main sequence stars, it is then possible to infer a distance of about 90 pc for the binary pair. For a spectral region shortward of the Lyman limit at $912 \AA$ there is very strong absorption in the interstellar medium by bound-free transitions of neutral hydrogen. The detection of any EUV flux from Feige 24 at this distance indicates that the local interstellar hydrogen is only about 0.01 to 0.02 particles per cubic centimetre, very low compared with the mean value for the Galactic plane of about 0.6 particles per cubic centimetre. This reinforces the view that the interstellar medium is very clumpy, with strong concentrations into clouds coexisting with a tenuous intercloud medium.

It has been suggested that Feige 24 shows the spectral characteristics of old novae, in which accretion of gas from the red dwarf across onto the white dwarf has liberated radiation. If such processes are still occurring, the resulting radiation is liable to be variable, and the continued use of Feige 24 as a spectrophotometric standard might justifiably be questioned.

\title{
Effects of photon momentum
}

\section{from a Correspondent}

THE photon dynamics resulting from light absorption by an atom are sufficient to cause an observable deflection of the excited atom. Radiation effects of this nature can now be utilised as techniques in atomic scattering experiments opening up a new and exciting area of investigation.

The advent of commercially available tunable continuous wave $(\mathrm{CW})$ dye laser systems now enables atomic physicists selectively to prepare the target atom in a scattering experiment in a well defined excited atomic state. The tunable power currently available (several milliwatts at a bandwidth of $5 \mathrm{MHz}$ ) is sufficient to maintain the necessary excited state population required for a scattering experiment to be performed. The sodium atom with its resonance transition $3^{2} \mathrm{~S}$ $3^{2} \mathrm{P}$, suitably placed in the region of efficient organic dye operation is a very attractive candidate for optical pumping. Work on the scattering of state selected sodium atoms $\left(3^{2} \mathrm{P}_{3 / 2}\right)$ off neon atoms has been reported (Carter et al., Phys. Rev. Lett. 35, 1144; 1975) in the determination of ground and excited potentials of the NaNe molecules. Hertel et al. ( $J$. Phys. B. 7, 570; 1974) were the first to perform high resolution electron scattering experiments on laser-excited sodium atoms where elastic, inelastic and superelastic processes were studied.

In the work by Hertel only differential cross section $\sigma(\theta)$ measurements were made where the scattered electron was monitored over a limited angular scattering range $\theta$. To establish a total cross section, measurement of $\sigma(\theta)$ is required over the angular range $\theta=0$ to $180^{\circ}$. An alternative method for directly measuring the total cross section $\sigma$ for electron atom scattering, that is, atomic beam recoil, was developed several years ago (Bederson in Methods of Experimental Physics (ed. Bedersen B. and Fite W. L.) 7A, 89 (Academic, 1968) for measuring of or the scattering of electrons off ground state atoms. In this procedure a well defined narrow atomic beam is cross fired at right angles by a beam of low energy electrons. The collimation in the apparatus after the interaction region is sufficient to enable the reduction in flux of the atomic beam to be monitored. The signal scattered out of the incident beam by the electrons $\Delta I=I_{o}-I_{\mathrm{s}}$ (where $I_{\mathrm{o}}$ and $I_{\mathrm{s}}$ are the atomic beam current with the electron beam off and on respectively) therefore gives a direct measure of the total electron scattering cross section. The removal of atoms from the beam is the dynamical effect of the momentum transfer imparted by the scattered electron to the atoms and this is sufficient to cause atomic recoils of several degrees.

No scattering experiments so far have taken advantage of the dynamical effects of photon-atom interactions as a diagnostic method. The momentum transferred to an atom, of mass $m$ and velocity $V$, by resonance absorptions, at wavelength $\lambda$, will result in a recoil through an angle $\alpha_{0}(=h / m V \lambda)$ with a recoil in the opposite direction for stimulated emission. Since spontaneous emission is random in direction the steady state recoil experienced by an atom passing through a laser beam will saturate at a value of $\alpha_{0} t / 2 \tau$, where $t$ is the time spent in the laser beam and $\tau$ is the natural life time of the atomic state being excited. In the experiment of Bhaskar et al. (Phys. Rev. Lett. 38, 14; 1977) $\alpha_{0} \approx 3 \times 10^{-5} \mathrm{rad}$ per photon absorption and the nett atomic beam deflection observed at $80 \mathrm{~cm}$ from the interaction region is several millimetres. This photon dynamical effect can now be combined with the atom recoil technique where the laser beam is perpendicular to the plane containing the atom and electron beams. This will yield the total cross section for scattering of electrons from the excited state $3^{2} \mathrm{P}_{3 / 2}$ $\left(M_{\mathrm{F}}=3\right)$ using the relation

$$
\left.\Delta I_{\text {on }} / \Delta I_{\text {off }}=f\left(\sigma_{\mathrm{c}} / \sigma_{\mathrm{g}}\right)-1\right)+1
$$

where $\Delta I_{\mathrm{on}}$ and $\Delta I_{\text {orr }}$ are the atom scattering out signals with the laser beam on and off respectively, and $\sigma_{\mathrm{e}}$ and $\sigma_{\mathrm{g}}$ are the total cross sections for electron scattering off excited and ground state atoms respectively. Bhaskar et al. have measured the absolute value of $\sigma_{e}$ at an incident electron energy of $4.4 \mathrm{eV}$ and obtained a value of $(285 \pm 55) \times 10^{-16} \mathrm{~cm}^{2}$.

Extension of this technique to differential cross-section measurements and to experiments in which the spin state of the atom is selected will greatly increase the knowledge of fundamental procesess in atomic scattering.

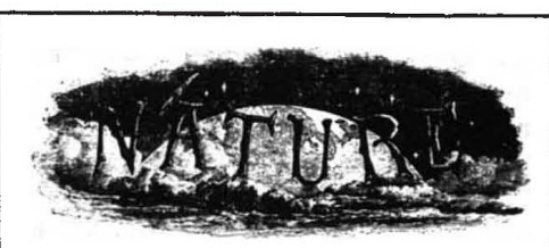

\section{A hundred years ago}

A MEDAL to commemorate the part taken by the Institute of France in the observation of the transit of Venus has been struck at the national mint. It bears the representation of a female passing before the car of Apollo, with the motto in Latin, "Quo distent spatio, sidera juncta docent." Each member of the Institute has received a silver medal, as well as the heads of the mission; the assistants received a bronze one. A medal has been cast in gold and presented to $M$. Dumas, the President of the Transit Commission. The expenses were defrayed by subscription among the members of the Institute.

From Nature 15, February 15, 344; 1877. 